\title{
Cone formation on Ag surface bombarded by Ar at oblique incidence
}

\author{
S R BHATTACHARYYA, T K CHINI ${ }^{\dagger}$ and D BASU \\ Saha Institute of Nuclear Physics, Block-AF, Sector 1, Bidhan Nagar, Calcutta 700064, India \\ 'Present address: Department of System Engineering, Nagoya Institute of Technology, \\ Gokiso-cho, Showa-ku, Nagoya 466, Japan
}

MS received 13 January 1995; revised 4 April 1995

\begin{abstract}
Sputter-induced topographical modifications of a silver surface bombarded by mass-analysed ions of $30 \mathrm{keV}^{40} \mathrm{Ar}^{+}$obtained from an electromagnetic isotope separator incident at an angle of $58^{\circ}$ was studied. Analysis of the silver surface by scanning electron microscopy showed that most of the bombarded area was covered with features similar to corrugated terrace steps, sharp and fine cones, and pyramids. The underlying mechanism for formation of such features is discussed. The method of formation of such sharp cones produced by ion bombardment will increasingly find applications in the formation of fine tips required in scanning probe and field emission microscopy.
\end{abstract}

Keywords. Sputter-erosion; sputter-induced cones; topography; oblique cone formation.

\section{Introduction}

An interesting aspect of heavy-ion irradiation of the surfaces of solids at relatively high doses $\left(>10^{17}\right.$ ions $\left./ \mathrm{cm}^{2}\right)$ is the development of well-defined topography which has been the subject of experimental and theoretical investigations in view of both the fundamental physical concept and substantial technological interest. These features due to sputtering often resemble cones, pyramids, steps, ripples, etc. As far as applications are concerned (Auciello 1982), a textured surface due to sputtering can be used as cathode for better field emission or explosion emission, in experiments requiring low electron or ion reflectance, to increase optical absorption important in solar cell technology, and to increase secondary emission. It is also established that textured surface made by sputtering could be beneficial in lowering the surface erosion by energetic ions striking the first walls of controlled fusion reactors. These applications show that studies of surface topography of ion-bombarded solids are important for better understanding of the physical processes in the evolution of such features. With the advent of scanning electron microscopy it has been possible to investigate solid surfaces in greater detail and precision. It has been found that the nature and form of surface features are related to the ion species, the material under study and the environment in the collision chamber. Therefore the mass, energy, dose and current density of the projectile ion and the mass, orientation and environmental conditions of the target material are crucial in determining the nature and form of topographical features developed (Carter et al 1983). Sputtered erosion by ion bombardment is the most effective and controlled way of processing sharp and fine tips which are essential in scanning probe and field emission microscopy. Quite recently, computer simulation studies have been started (Wege et al 1993) to see how ion-beam sputtering can be used to construct fine tips with small apex angle and radius as applied to the above mentioned microscopy. 
In this report we show evidence of topography consisting mostly of cones on mirror-polished silver samples bombarded by $30 \mathrm{keV}$ mass-analysed ${ }^{40} \mathrm{Ar}^{+}$ions at oblique incidence of $58^{\circ}$ with the normal to the surface. Silver was chosen as target because of its large secondary electron emission under SEM and therefore reveals greater details of the features developed on the surface. Preliminary results on development of fine and sharp cones or tips by ion-induced sputter erosion at oblique incidence are also reported.

\section{Experimental}

Samples of polycrystalline $\mathrm{Ag}$ were bombarded at oblique incidence of $58^{\circ}$ with surface normal by $30 \mathrm{keV}$ mass-analysed ions of ${ }^{40} \mathrm{Ar}^{+}$obtained from an electromagnetic isotope separator. The residual gas pressure in the target chamber was around $2 \times 10^{-5}$ torr. Beam current density was around $200 \mu \mathrm{A} / \mathrm{cm}^{2}$. Bombardment doses were measured by integrating the current on the target by a current integrator (Danfysik 554). To suppress secondary electrons, the collector cups were biased with negative potential with respect to the target. The surfaces were bombarded with a dose of $1 \times 10^{19}$ ions $/ \mathrm{cm}^{2}$ and were examined by a Hitachi S-415S scanning electron microscope.

\section{Results and discussion}

\subsection{Observation of surface under SEM}

The micrographs (figure 1) of $\mathrm{Ag}$ surface bombarded by $30 \mathrm{keV} \mathrm{Ar}^{+}$projectile at an oblique incidence of $58^{\circ}$ with the surface normal show interesting features. Most of the bombarded areas are covered with features similar to corrugated terrace steps (figure 1a) and sharp cones (figures $1 \mathrm{~b}-\mathrm{d}$ ). These terrace planes are always aligned parallel to the ion beam. Faceted cones or pyramids developed are also aligned with their axes parallel to the ion beam direction as seen in figures $1 \mathrm{~b}$ and $1 \mathrm{c}$. Some of the cones are very sharp; the apex angles of these cones range from $11^{\circ}$ to $17^{\circ}$. The important observation in the present case is that some of the oblique cones formed are bent at their stems or tips. A typical case is shown in figure 1d.

\subsection{Possible mechanism of cone formation on the sample}

According to first-order erosion theory, the dependence of sputtering yield on angle of incidence of the ion beam plays a major role in formation of sputtered cones, as suggested by different authors (Carter et al 1983; Ghose and Karmohapatro 1990). If $\theta$ be the angle between the direction of the incident ion beam and the normal to the surface, then the sputtering yield $Y(\theta)$ follows a $\sec \theta$ dependence when $\theta$ is low. However, $Y(\theta)$ deviates from $\sec \theta$ dependence for large values of $\theta$, reaches a maximum at $\hat{\theta}$ between $60^{\circ}$ and $80^{\circ}$ and then falls to zero at $\theta=90^{\circ}$. This variation of $Y(\theta)$ with $\theta$ is shown in figure 2. A number of authors have discussed the mechanism of cone formation as summarized by Ghose and Karmohapatro (1990) in their recent review of the subject. The following mechanism for the nucleation of cones can be regarded as responsible in our present investigation. 
(i) During sputtering by energetic ions, an impurity of lower sputtering yield than the matrix causes erosion discontinuity on the surface which subsequently takes a conical shape due to the angular variation of sputtering yield.

(ii) The surface impurity seems to be the initiator and results in the formation of cones by a combination of erosion and surface migration.

\subsection{Mechanism of oblique cone formation}

The underlying mechanism for oblique cone or pyramid formation is found to be similar to that responsible for the formation of normal cones or pyramids. However, Auciello and Kelly (1982) identified five sources of perturbation to the first-order erosion theory to account for the evolution of oblique cones. These are the growth of topography relative to the instantaneous surface, asymmetrical flux enhancement originating
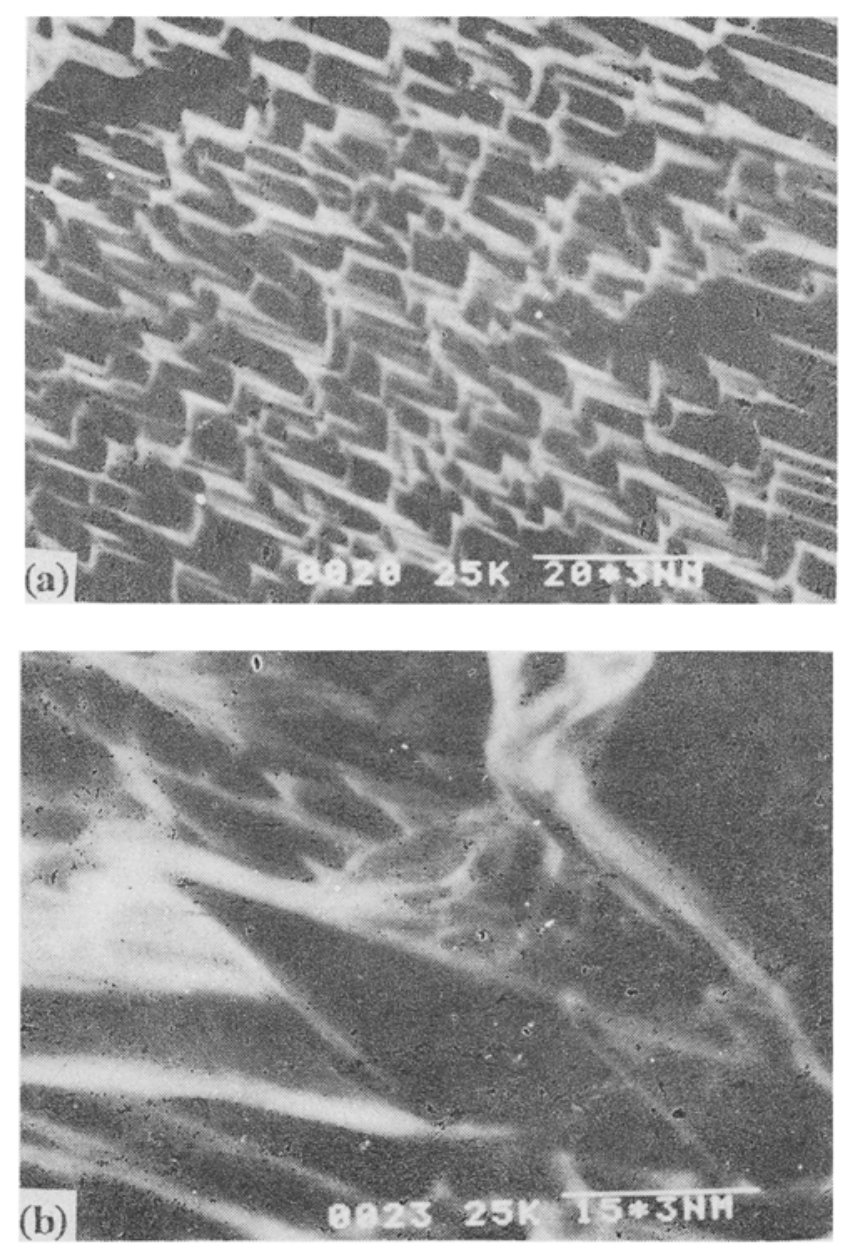

Figure 1. a-b. 

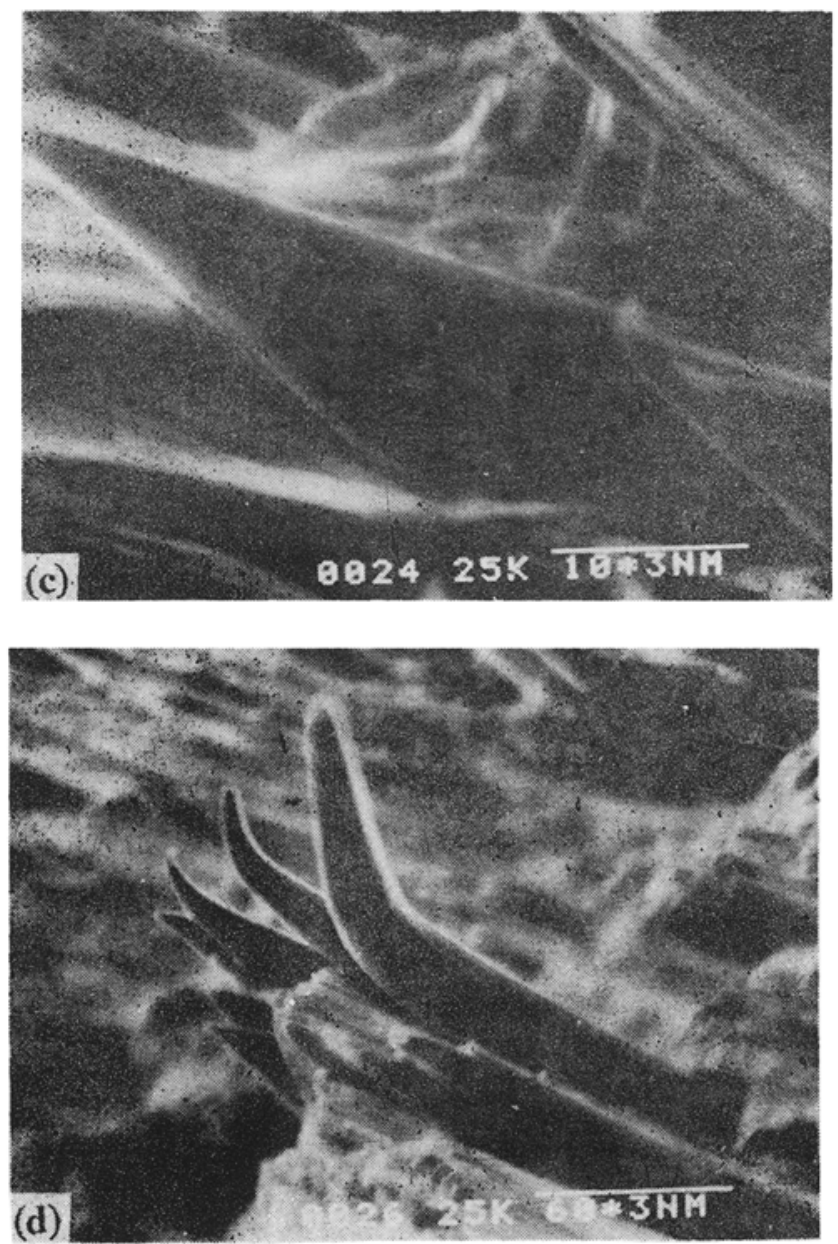

Figure 1. Scanning electron micrographs of Ag surface bombarded by $30 \mathrm{keV}^{40} \mathrm{Ar}^{+}$ions at an oblique incidence of $58^{\circ}$ with a dose of $1 \times 10^{19}$ ions $/ \mathrm{cm}^{2}$. (a) Corrugated faceted structure at the central part of the bombarded spot. (b and c) Oblique cones along the ion beam direction are seen. (d) Sharp cones bent at their stems are seen. Tilt angle of the microscope was $30^{\circ}$ in a and $60^{\circ}$ in b, c and d.

from the high-angle surface, redeposition of sputtered material, effects due to beam divergence, and the role of latent planes. Among these sources, the effects of beam divergence, redeposition and latent plane have important contributions to such development. At lower doses of oblique incidence or at early stages of erosion, a rippled or corrugated structure may form. Quasiperiodic structures like those in figure 1a have been observed on Ge (Chini et al 1992) and Si (Chini et al 1991). In the present studies, however, no ridge or web-like structures were found. Careful observation also shows that the oblique and bent cones in figure 1d have their diameters almost uniform along the length and they are somewhat sharper in appearance in contrast to other oblique cones (figures $1 \mathrm{~b}, \mathrm{c}$ ). 


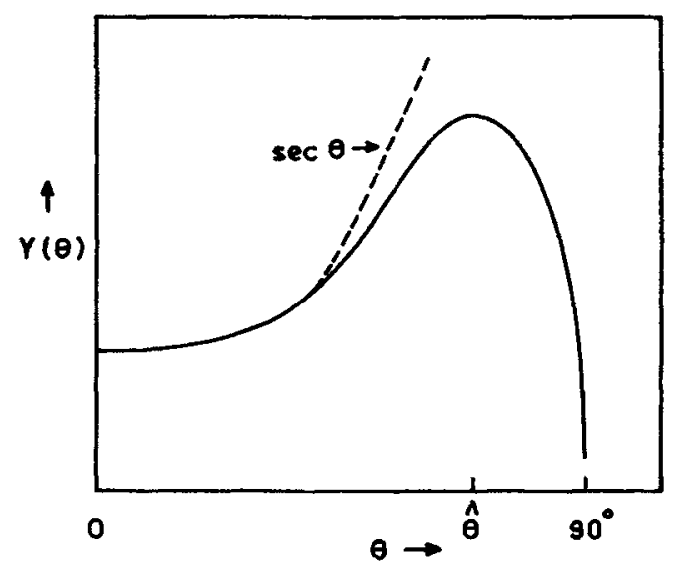

Figure 2. Variation of sputtering yield $Y(\theta)$ as a function of ion incident angle $\theta$.

\subsection{Bent cones}

Some cones developed on silver in the present studies are found bent at their tips or stems. Such spontaneous bending of cones is rare in cone evolution on polycrystalline metal targets. The mechanism of bending of the oblique cones in the present case may be conceived of as due to stresses developed on these cones in the process of beam divergence or fluctuation away from the cone axes. According to Wilson (1989), around the tip of a typical $\hat{\theta}$ cone, the surface stress exceeds the critical shear stress of a metal and, therefore, if the orientation is such that there is an asymmetry in resolved shear stresses along low index planes, plastic deformation can occur resulting in the bending of some cones in a direction where yield strength is minimum.

\section{Conclusion}

Preliminary studies of topography of Ag surface were carried out with ${ }^{40} \mathrm{Ar}^{+}$ions incident at an oblique angle. The modified topography shows sharp oblique cones and corrugated features. The formation of oblique cones seems not to be the consequence of the first-order erosion theory alone. Therefore the angle-dependent topographical evolution and thereby various conditions for fine cone formation as feeding data are essential to optimize the required results. Studies on the same aspect may be pursued both theoretically and experimentally at different angles of ion incidence for better understanding of oblique cone formation, and the results can be used to process fine and sharp tips as used in scanning probe or field emission microscopes.

\section{Acknowledgement}

The authors thank Electron Microscope Facility of the Indian Association for the Cultivation of Science, Calcutta for SEM work. 


\section{References}

Auciello O 1982 J. Vac. Sci. \& Technol. 19841

Auciello $O$ and Kelly R 1982 Radiat. Eff. 66195

Carter G, Navinsyek $\mathbf{B}$ and Whitton J L 1983 in Sputtering by particle bombardment II (ed.) R Behrisch (Berlin: Springer) p. 231

Chini T K, Bhattacharyya S R, Ghose D and Basu D 1991 Jpn. J. Appl. Phys. 302895

Chini T K, Bhattacharyya S R, Ghose D and Basu D 1992 Vacuum 43219

Ghose D and Karmohapatro S B 1990 Adv. Electron. Electron Phys. 7973

Wege S, Bautsch M, Rübesame D, Niedrig H and Wittich T 1993 Nucl. Instrum. Methods B80 94

Wilson $1 \mathrm{H} 1989$ Surface Topography 289 